\title{
Review of: "Control of protein synthesis and memory by GluN3A-NMDA receptors through inhibition of GIT1/mTORC1 assembly"
}

Kasia Radwanska

Potential competing interests: The author(s) declared that no potential competing interests exist.

The authors show that Grin3A subunit of NMDAR receptor controls synaptic activation and localisation of mTOR pathway by competing interactions with GIT1 protein. They show that removal of Glun3A allows for GIT1/mTOR interactions that potentiate mTOR-dependent protein synthesis. Grin3a KO mice show also enhanced memory in several tasks without impairments of memory flexibility and extinction. This is a very exciting line of research as it focuses on poorly understood non-ionotropic functions of NMDARs. Although, overall this is a very comprehensive study some points still remain unclear.

1. The authors propose that GluN3A modules the assembly of synaptic GIT1/mTORC1 complexes. However, they do not deliver the final proof that GIT1-regulated mTORC1 activation is specific for synapses. Figure 4C shows that GIT1/mTORC1 complexes also exist in dendrites. Sensitivity to APV and MK-801 indicates its dependence on NMDAR receptors, which can be, however, extra-synaptic. Inhibition of Fos protein expression by rapamycin in this model actually proves that it also regulates non-synaptic translation. Along this line, if the authors want to state that GIT1/GIuN3A interaction controls mTOR localisation in a dendritic spine/synapse the in situ proximity ligation assay should be quantified (Fig. 4C) to indicate what fraction of the puncta is localised in dendritic spines vs dendrites vs soma. Optimally, this analysis should include WT and GluN3-/- neurons, and stimulated vs control cultures.

2. Behavioural experiments: The authors attempt to link the GluN3A/mTOR-regulated translation in cortical and hippocampal neurons with cognitive enhancements in three behavioural tests (MWM, CTA and FC). It is, however, uncertain how the authors imagine the link between GluN3A and memory mechanisms. The functional interactions between Grin3a-/- mutation and training should be shown in those regions. Are there any changes of synaptic efficacy, neuronal excitability or dendritic spines dynamics regulated by memory AND Glun3A? It would be also interesting to know in which brain regions GluN3A contributes to memory modulation. Is GIuN3A/GIT1/mTORC1 interaction regulated during memory formation and extinction?

\section{Statistics:}

- The statistics of all tests is missing; 
- The exact $p$ values should be given;

- The choices of particular tests should be justified (normal vs other distributions?)

M\&M mention only cortical cultures so the protocols for the hippocampal cultures should be added. 